\title{
Rentabilidade econômica de unidades operacionais de uma rede logística: novo método de cálculo
}

\author{
Reinaldo Pacheco da Costa ${ }^{1}$; André Ambrosio Abramczuk²; \\ Hugo Tsugunobu Yoshida Yoshizaki ${ }^{3}$; Hugo Yasuyuki Tsukamoto ${ }^{4}$; Davi Claudio ${ }^{5}$
}

\begin{abstract}
Resumo: Este artigo apresenta um método de cálculo de rentabilidade econômica de unidades operacionais (UO's) de empresas de transporte de carga fracionada que operam através de uma rede logística com múltiplas UO's. O cálculo da rentabilidade econômica de cada uma destas UO's é um problema complexo. Devido ao intercâmbio de cargas entre UO's, as receitas de uma UO estão interligadas com as receitas de outras UO's. Os métodos tradicionais de contabilização enfrentam dificuldades quando empregados para determinar a rentabilidade econômica de UO's de uma rede logística de UO's interdependentes. O método descrito neste artigo elimina estas dificuldades por meio do conceito de margem de contribuição.
\end{abstract}

\begin{abstract}
This paper offers a method for evaluating the profitability of operation units (OS) of less-than-truckload (LTL) carriers conveying shipments through a network with multiple OS. The evaluation of the profitability of each one of these OS is a complex problem. Due to exchanges of shipments between OS, the revenues of one OS are intertwined with the revenues of other OS. Traditional accounting methods show themselves cumbersome when employed to evaluate the profitability of OS of a logistical network of interdependent OS. The method described in this paper overcomes these difficulties by means of the concept of contribution margin.
\end{abstract}

\section{INTRODUÇÃO}

Para coletar, armazenar e distribuir mercadorias, uma empresa de transporte pode utilizar instalações próprias, contratar serviços de terceiros, fazer parcerias operacionais com outras empresas de transporte ou combinar todas estas alternativas. Para avaliar as alternativas quanto à adequação aos objetivos de assegurar rentabilidade econômica para a empresa e qualidade dos serviços prestados aos clientes, devem ser consideradas no mínimo quatro variáveis: nível da qualidade dos serviços que se pretende prestar, localização das UO's, rotas de transporte e volumes de carga movimentados numa UO e entre UO's. Estas variáveis são interdependentes, razão por que a avaliação das alternativas é um problema complexo.

Crença comum em diversos ramos de negócios é a de que uma empresa deve possuir vários armazéns locais próximos aos clientes, como forma de prestar pronto atendimento aos pedidos destes de um lado e, de outro, compensar deficiências de transporte e de processamento de pedidos (Wanke, 2001). Inovações

\footnotetext{
${ }^{1}$ Reinaldo Pacheco da Costa, Escola Politécnica da Universidade de São Paulo. São Paulo, SP, Brasil. (e-mail: rpcosta@usp.br).

${ }^{2}$ André Ambrosio Abramczuk, Escola Politécnica da Universidade de São Paulo. São Paulo, SP, Brasil. (e-mail: bramczuk@usp.br).

${ }^{3}$ Hugo Tsugunobu Yoshida Yoshizaki, Escola Politécnica da Universidade de São Paulo. São Paulo, SP, Brasil. (e-mail: hugo@usp.br).

${ }^{4}$ Hugo Yasuyuki Tsukamoto, Escola Politécnica da Universidade de São Paulo. São Paulo, SP, Brasil. (e-mail: hugoyasuyuki@yahoo.com.br).

${ }^{5}$ Davi Claudio, Escola Politécnica da Universidade de São Paulo. São Paulo, SP, Brasil. (e-mail: dclaudio@uol.com.br).
}

Este artigo é parte de TRANSPORTES, volume XIV, número 2, dezembro de 2006. ISSN: 1415-7713. no sistema de transporte - monitoramento via satélite de veículos em trânsito, melhoria de confiabilidade no planejamento de seus horários de chegada a uma localidade e saída dela etc. - e na tecnologia de informação (com redução dos tempos de transmissão e processamento de informações) contribuíram para atenuar e em alguns casos virtualmente eliminar estas deficiências, permitindo reduzir o número de UO's necessárias para atender em tempo hábil aos pedidos de clientes de determinada área. Muitas empresas, contudo, aumentaram de maneira desordenada o número de UO's próprias, geralmente posicionando-as em localizações inadequadas. Grande número de instalações não significa necessariamente nível adequado da qualidade dos serviços prestados aos clientes; se a cadeia logística não funciona de maneira eficiente, contribui somente para tornar elevados os custos de operação.

A exigência de serviços de transporte com qualidade e com baixo custo e o ingresso de grandes operadores logísticos (inclusive internacionais) no mercado brasileiro tornam necessidade premente a reestruturação da gestão da rede logística das empresas de transporte, se quiserem ser competitivas e rentáveis. Isto exige, entre outras medidas, determinar quais UO's manter de maneira economicamente rentável e quais UO's desativar sem prejudicar o nível da qualidade dos serviços prestados aos clientes. A questão fundamental para a qual buscar resposta é como definir a viabilidade econômica e estratégica das UO's. A resposta exige que se disponha de um método de análise da rentabilidade das UO's, o que por sua vez exige critérios de quantificação de custos e receitas de cada UO isoladamente. Buscar esta resposta é buscar solução para o problema de múltiplas UO's. Este envolve 
três aspectos:

1. Dada uma configuração existente de UO's, qual a rentabilidade econômica de cada uma delas isoladamente, sabendo-se que cada UO pode fazer coleta e distribuição de cargas, receber cargas de outras UO's e remeter cargas para elas, para distribuição ou novos transbordos?

2. Dada uma configuração existente de UO's e suas rentabilidades econômicas, qual a reconfiguração que proporciona melhoria da rentabilidade econômica da rede logística como um todo? Em outros termos: dada uma configuração existente de UO's, quais as localizações onde manter uma UO, quais as localizações cuja UO desativar, para assegurar melhoria da rentabilidade econômica da rede logística?

3. Havendo possibilidade de alterar a quantidade de UO's e suas localizações, quantas UO's instalar e onde, para assegurar a melhor rentabilidade possível da rede logística?

O problema (3) é um problema de criação de uma rede logística, cuja solução consiste em escolher a mais adequada dentre todas as alternativas possíveis de configuração da rede logística. A quantidade de alternativas cresce exponencialmente com o número de localizações possíveis. Dadas, por exemplo, duas localizações $-A$ e $B$ - são possíveis três configurações da rede logística: instalar uma UO somente em $A$; instalar uma UO somente em $B$ e; instalar UO's em $A$ e $B$. Dadas três possíveis localizações, haverá sete alternativas. Se uma empresa tiver que considerar oito possíveis localizações de UO's, o número de alternativas de configuração da rede logística será $255\left(=2^{8}-1\right)$.

Os métodos empregados para resolver o problema (3) são todos métodos ex ante, não se prestando para resolver os problemas (1) e (2), que são problemas ex post. Para a solução destes problemas apresenta-se a seguir um novo método.

\section{O NOVO MÉTODO}

Os métodos tradicionais de análise de rentabilidade econômica tratam os problemas (1) e (2) da seguinte maneira, em linhas gerais: (a) Consideram que uma UO gera receitas com a coleta de cargas. Desta maneira, UO's que recebem para distribuição cargas transferidas de outras UO's em volume maior do que cargas que coletam invariavelmente apresentam rentabilidade baixa ou negativa; (b) Fazem rateio dos custos da UO sobre a carga a jusante. Ambos estes procedimentos distorcem a rentabilidade das UO's. O termo 'rateio' implica o uso do método de custeio por absorção (também conhecido como método de custeio integral). Este método é sobejamente acusado de distorcer o cálculo de rentabilidade de produtos industrializados
(Kaplan \& Atkinson, 1989; Martins, 1990). Não é diferente quando aplicado ao cálculo de rentabilidade econômica das UO's de uma empresa de transporte com múltiplas UO's.

Com a finalidade de eliminar estas distorções, Costa (2002) propõe um novo método de cálculo da rentabilidade econômica de UO's de uma rede logística fundamentado no conceito de margem de contribuição.

No novo método, o cálculo da rentabilidade econômica das UO's isoladamente, e da rede logística como um todo, fundamenta-se numa concepção de balanço de carga das UO's e numa concepção própria de atribuição de custos e de receitas às UO's. Por isto, a seguir apresenta-se primeiramente a concepção de balanço de carga das UO's e, com fundamento nesta, as equações de cálculo da rentabilidade econômica. Para as equações adotam-se as seguintes convenções:

a. Todas as grandezas são representadas por letras maiúsculas. Quando uma grandeza é representada por um grupamento de letras, o grupamento é colocado entre parênteses.

b. Um sub-índice associa uma grandeza a uma UO.

c. Pares de sub-índices indicam transferência entre UO's. O primeiro sub-índice indica a UO de origem, o segundo sub-índice indica a UO de destino. Para evitar equívocos, os sub-índices de um par são separados por vírgula. Por exemplo, $(V T)_{i, j}$ representa volume de carga transferido da UO identificada pelo sub-índice $i$ para a UO identificada pelo sub-índice $j,(V T)_{k, m}$ indica volume da carga transferida de $\mathrm{UO}_{k}$ para $\mathrm{UO}_{m}$.

d. Para evitar proliferação do símbolo $\Sigma$, adota-se a seguinte convenção de soma: dado um subíndice $k$ em relação ao qual se deve fazer uma somatória de $n$ termos (isto é, com $k=1,2,3, \ldots$, $n$ ), o sub-índice $k$ é colocado entre parênteses e, quando necessário, especificado o número de termos presentes na soma. Assim, $C_{(k)}$ com $1 \leq k \leq n$ significa uma soma de $n$ elementos:

$$
C_{(k)}=C_{1}+C_{2}+C_{3}+C_{4}+\ldots+C_{n}
$$

No caso de pares de sub-índices, a convenção de soma será usada da seguinte maneira:

$$
(C T)_{i,(j)}=(C T)_{i, 1}+\ldots+(C T)_{i, n}
$$

Por causa da natureza do problema a que se referem, nas equações presentes neste artigo de modo geral numa soma de termos tem-se $(K G)_{i, k}=0$ para $i=$ $k$; ocorrendo a condição de $(K G)_{i, k} \neq 0$ para $i=k$, isto será ressaltado. Em alguns casos, a condição de $(K G)_{i, k}=0$ para $i=k$ será posta em evidência escrevendo-se 


$$
(C T)_{i,(k)} \quad i \neq k
$$

No caso em que a soma envolva ambos os subíndices de um par, a representação e correspondente significado são:

$$
(C F)_{(i, j)}=(C F)_{(i), 1}+(C F)_{(i), 2}+\ldots+(C F)_{(i), n}
$$

\subsection{Balanço de carga}

Para quantificar a carga, considera-se o volume expresso numa única unidade de medida válida para toda a rede logística: metros cúbicos, quilogramas, toneladas etc.

$\mathrm{O}$ volume total de carga movimentada numa $\mathrm{UO}$ em determinado intervalo de tempo obedece ao princípio de conservação de carga: o volume total de carga que entra numa UO é igual ao volume total de carga que sai da UO.

A seguir usam-se as seguintes representações:

$(V E)_{i}$ : volume total de carga que entra na $\mathrm{UO}_{i}$

$(V S)_{i}$ : volume total de carga que sai da $\mathrm{UO}_{i}$

$(V C)_{i}$ : volume total de carga coletada pela $\mathrm{UO}_{i}$

$(V D)_{i}$ : volume total de carga distribuída pela $\mathrm{UO}_{i}$

$(V T)_{i, j}$ : volume de carga transferida pela $\mathrm{UO}_{i}$ para a $\mathrm{UO}_{j}$

$(V T)_{k, i}$ : volume de carga recebida pela $\mathrm{UO}_{\mathrm{i}}$ para ela transferida pela $\mathrm{UO}_{\mathrm{k}}$

$\mathrm{O}$ volume total de carga transferida pela $\mathrm{UO}_{i}$ para outras UO's é dado por

$$
(V T)_{i,(j)} \quad i \neq j
$$

$\mathrm{O}$ volume total de carga recebida pela $\mathrm{UO}_{i}$ para ela transferida por outras UO's é dado por

$$
(V T)_{(k), i} \quad i \neq k
$$

Pelo princípio de conservação de carga, numa UO tem-se:

$$
\begin{aligned}
& (V E)_{i}=(V C)_{i}+(V T)_{(k), i} \quad i \neq k \\
& (V S)_{i}=(V D)_{i}+(V T)_{(i), j} \quad i \neq j
\end{aligned}
$$

Subtraindo membro a membro a equação (2) da equação (1), o princípio de conservação de carga numa UO é expresso por

$$
(V C)_{i}+(V T)_{(k), i}-(V D)_{i}+(V T)_{(i), j}=0
$$

Dada uma rede logística constituída por n UO's, a equação (3) aplica-se a cada uma delas; disto resulta um sistema de $n$ equações que, somadas membro a membro, dão como resultado:

$$
(V C)_{k}-(V D)_{k}=0 \quad 1 \leq k \leq n
$$

Qualquer um dos termos da equação (3) pode isoladamente ser nulo. Se há cargas entrando numa UO e saindo dela - isto é, $(V E)_{i} \neq 0$ e $(V S)_{i} \neq 0$ - é óbvio que nunca se pode ter simultaneamente $(V C)_{i}=$ $(V T)_{(k), i}=0$ nem $(V D)_{i}=(V T)_{i,(j)}=0$, mas as seguintes combinações são possíveis:

$$
\begin{aligned}
& (V C)_{i}=(V D)_{i}=0 \Rightarrow(V T)_{(k), i}=(V T)_{i,(j)} \\
& (V C)_{i}=(V T)_{i,(j)}=0 \Rightarrow(V T)_{(k), i}=(V D)_{i} \\
& (V T)_{(k), i}=(V D)_{i}=0 \Rightarrow(V C)_{i}=(V T)_{i,(j)} \\
& (V T)_{(k), i}=(V T)_{i,(j)}=0 \Rightarrow(V C)_{i}=(V D)_{i}
\end{aligned}
$$

A condição (4) identifica uma UO essencialmente de transbordo.

A condição (5) identifica uma UO essencialmente de distribuição de cargas recebidas de outras UO's.

A condição (6) identifica uma UO que coleta cargas e transfere integralmente o volume coletado para outras UO's.

A condição (7) identifica uma UO que não interage com outras UO's da rede logística, pois não recebe cargas de outras UO's nem lhes transfere cargas; é uma UO autística.

De interesse para o método de calculo de rentabilidade econômica das UO's que se apresenta a seguir é o fator de transferência de margem de contribuição de uma UO para outra, dado por:

$$
\Phi_{j, i}=\frac{(V T)_{i, j}}{(V T)_{(i), j}}
$$

isto é, o fator de transferência da margem de contribuição primária da unidade $j$ para a unidade $i$ (que transferiu carga para a unidade $j$ ) é a relação entre o volume de carga transferido da unidade $i$ para a unidade $j$ e o volume total de cargas transferidas por outras unidades para a unidade $j$.

Com isto, vamos ter sempre que

$$
\Phi_{j,(i)}=1
$$

Ou seja, uma unidade operacional $\mathrm{j}$ vai sempre transferir integralmente sua margem de contribuição primária para as unidades das quais tenha recebido carga por transferência, independentemente da relação entre volume de carga recebida e volume de carga coletada e carga distribuída. Se, por exemplo, uma unidade operacional coleta 390 unidades de carga, receba 10 unidades de carga de outra unidade operacional, distribui 300 unidades de carga e transfere 100, vai transferir para a unidade operacional da qual recebeu 10 unidades de carga $100 \%$ da sua margem de contribuição primária. 


\subsection{As equações do novo método}

O cálculo da rentabilidade econômica das UO's de uma rede logística preconizado pelo novo método fundamenta-se nas seguintes regras de procedimento:

1. A receita de uma UO é gerada somente pelas cargas distribuídas pela UO.

Esta regra é oposta à regra adotada pelos métodos tradicionais, que contabilizam a receita de uma UO em função da carga por ela coletada .

Nas equações a seguir, $R_{i}$ representa a receita de uma $\mathrm{UO}_{i}$.

2. Consideram-se no cálculo somente os custos operacionais da UO:

$(C P)_{i}$ : custos próprios da $\mathrm{UO}_{i}$

= custos fixos + custos de coleta de cargas + custos de distribuição de cargas;

$(C T)_{i, j}$ : custo de transferência de carga, em que a UOi incorre com a transferência que faz de carga para uma UOj.

3. A margem de contribuição primária de uma UO é, por definição:

$$
(M C P)_{i}=R_{i}-(C P)_{i}
$$

4. Toda $U O_{i}$ que recebe cargas de uma UO $\mathrm{U}_{j}$ transfere para esta uma fração de sua margem de contribuição primária, fração esta determinada por:

$$
(M C T)_{i, j}=\Phi_{i, j} \cdot(M C P)_{i}
$$

5. Toda $U_{i}$ que transferiu cargas para uma $U O_{j}$ recebe desta uma fração da respectiva margem de contribuição primária. Desta fração, a $U O_{i}$ que transferiu carga para $U O_{j}$ deve deduzir o correspondente custo de transferência:

$$
(M C T)_{j, i}-(C T)_{i, j}=\Phi_{j, i} \cdot(M C P)_{j}-(C T)_{i, j}
$$

6. A margem de contribuição líquida de uma UOi é determinada por:

$$
\begin{gathered}
(M C L)_{i}=(M C P)_{i}-(M C T)_{i,(j)}+\ldots \\
\ldots .\left[(M C T)_{(j), i}-(C T)_{(i), j}\right]
\end{gathered}
$$

Substituindo nesta equação as grandezas correspondentes expressas pelas equações (8) e (9), obtém-se, finalmente:

$$
\begin{gathered}
\left.(M C L)_{i}=\left[R_{i}-(C P)_{i}\right] \cdot \mid 1-\Phi_{i,(j)}\right\rfloor+\ldots \\
\ldots .\left[(M C T)_{(j), i}-(C T)_{i,(j)}\right]
\end{gathered}
$$

Esta equação determina a rentabilidade econômica de uma UO como margem de contribuição da UO para a rentabilidade econômica da rede logística como um todo.

Dada uma rede logística constituída por $n$ UO's, a cada UO corresponde uma equação (12); a rede logís- tica é representada, portanto, por um sistema de $n$ equações lineares. A rentabilidade econômica da rede logística é dada pela margem de contribuição líquida total, isto é, por:

$$
(M C L)_{T O T A L}=(M C L)_{(i)} \quad 1 \leq i \leq n
$$

O resultado final é

$$
\begin{aligned}
& (M C L)_{\text {TOTAL }}=R_{(i)}-\left[(C P)_{i}-(C T)_{i}\right] \\
& \text { com } 1 \leq i \leq n, \quad 1 \leq j \leq n, \quad i \neq j
\end{aligned}
$$

\subsection{Verificação}

Para verificação simples do novo método, consideram-se três situações. As duas primeiras enquadram-se no problema (1), a terceira no problema (2).

\section{Situação 1:}

Uma rede logística é constituída por duas UO's, $\mathrm{UO}_{a}$ e $\mathrm{UO}_{b}$, ambas executando operações de coleta e distribuição de cargas e permuta de cargas entre si.

$\mathrm{Na} \mathrm{UO}_{a}$ tem-se, de acordo com a equação (8):

$$
(M C P)_{a}=R_{a}-(C P)_{a}
$$

Como recebe cargas transferidas de $\mathrm{UO}_{b}, \mathrm{UO}_{a}$ deve transferir para $\mathrm{UO}_{b}$ uma fração desta margem de contribuição primária:

$$
(M C T)_{a, b}=\Phi_{a, b} \cdot(M C P)_{a}
$$

De modo análogo, na $\mathrm{UO}_{b}$ tem-se:

$$
\begin{gathered}
(M C P)_{b}=R_{b}-(C P)_{b} \\
(M C T)_{a, b}=\Phi_{a, b} \cdot(M C P)_{a}
\end{gathered}
$$

As margens de contribuição líquida de cada uma das UO's são, pela equação (11):

$$
\begin{aligned}
& (M C L)_{a}=(M C P)_{a}-(M C T)_{a,(b)}+\left\lfloor(M C T)_{(b), a}-(C T)_{(a), b}\right\rfloor \\
& (M C L)_{a}=(M C P)_{a}-(M C T)_{a,(b)}+\left\lfloor(M C T)_{(b), a}-(C T)_{(a), b}\right\rfloor
\end{aligned}
$$

Somando estas equações membro a membro e fazendo as substituições adequadas, obtém-se:

$$
\begin{gathered}
(M C L)_{a}+(M C L)_{b}=R_{a}+R_{b}-\ldots \\
\quad \ldots\left[(C P)_{a}+(C P)_{b}+(C T)_{b, a}+(C T)_{a, b}\right]
\end{gathered}
$$

\section{Situação 2:}

Uma rede logística é constituída por três UO's, $\mathrm{UO}_{a}, \mathrm{UO}_{b}$ e $\mathrm{UO}_{c}$. $\mathrm{UO}_{b}$ é uma UO de transbordo; $\mathrm{UO}_{a}$ e $\mathrm{UO}_{c}$ fazem coleta e distribuição de cargas, transferem cargas para $\mathrm{UO}_{b}$ e dela recebem cargas. $\mathrm{UO}_{a} \mathrm{e}$ $\mathrm{UO}_{c}$ não fazem permuta de caras diretamente entre si, somente por intermédio de $\mathrm{UO}_{b}$. 
Posto isto, tem-se que as margens de contribuição primária das UO’s são:

$$
\begin{gathered}
(M C P)_{a}=R_{a}-(C P)_{a} \\
(M C P)_{b}=-(C P)_{b} \text { uma vez que } R_{b}=0 \\
(M C P)_{c}=R_{c}-(C P)_{c}
\end{gathered}
$$

As frações das margens de contribuição que as UO's transferem entre si são:

$$
\begin{aligned}
& (M C T)_{a, b}=\Phi_{a, b} \cdot(M C P)_{a} \\
& (M C T)_{b, c}=\Phi_{b, c} \cdot(M C P)_{b} \\
& (M C T)_{b, a}=\Phi_{b, a} \cdot(M C P)_{b} \\
& (M C T)_{c, b}=\Phi_{c, b} \cdot(M C P)_{b}
\end{aligned}
$$

A margem de contribuição líquida de cada uma das UO's é:

$$
\begin{gathered}
(M C L)_{a}=(M C P)_{a}-(M C T)_{a, b}+\left\lfloor(M C T)_{b, a}-(C T)_{a, b}\right\rfloor \\
(M C L)_{b}=(M C P)_{b}-(M C T)_{b, a}-(M C T)_{b, c}+\ldots \\
\ldots\left[(M C T)_{b, a}-(C T)_{a, b}\right]+\left[(M C T)_{c, b}-(C T)_{b, c}\right] \\
(M C L)_{c}=(M C P)_{c}-(M C T)_{c, b}+\left\lfloor(M C T)_{b, c}-(C T)_{c, b}\right\rfloor
\end{gathered}
$$

A margem de contribuição líquida de $\mathrm{UO}_{b}$ apresenta uma peculiaridade que deve ser ressaltada. Como $\mathrm{UO}_{b}$ é uma UO essencialmente de transbordo, não coletando cargas nem gerando receita própria por meio de distribuição de cargas, a equação correspondente à sua margem de contribuição líquida se traduz em

$$
\begin{array}{r}
(M C L)_{b}=\Phi_{a, b} \cdot(M C P)_{a}+\Phi_{c, b} \cdot(M C P)_{c}-\ldots \\
\ldots\left[(C T)_{b, c}+(C T)_{c, b}+(C P)_{c} \cdot\left(\Phi_{b, c}+\Phi_{a, b}\right)\right]
\end{array}
$$

Como $\mathrm{UO}_{b}$ é essencialmente de transbordo, a ela se aplica a condição (4). Pelo princípio de conservação de carga, tem-se, então:

$$
\left(\Phi_{b, c}+\Phi_{a, b}\right)=1
$$

Isto faz com que se tenha

$$
\begin{gathered}
(M C L)_{b}=\Phi_{a, b} \cdot(M C P)_{a}+\Phi_{c, b} \cdot(M C P)_{c}-\ldots \\
\quad \ldots\left[(C T)_{b, c}+(C T)_{c, b}+(C P)_{c}\right]
\end{gathered}
$$

Daí, o sistema de equações da rede logística pode ser escrito simplesmente de outra maneira:

$$
(M C L)_{a}=(M C P)_{a}-(M C T)_{a, b}+\left\lfloor(M C T)_{b, a}-(C T)_{a, b}\right\rfloor
$$

$$
\begin{aligned}
& (M C L)_{b}=\Phi_{a, b} \cdot(M C P)_{a}+\Phi_{c, b} \cdot(M C P)_{c}-\ldots \\
& \quad \ldots\left[(C T)_{b, c}+(C T)_{c, b}+(C P)_{c}\right]
\end{aligned}
$$

$$
(M C L)_{c}=(M C P)_{c}-(M C T)_{c, b}+\left\lfloor(M C T)_{b, c}-(C T)_{c, b}\right\rfloor
$$

Uma vez que $\Phi_{a, b} \cdot(M C P)_{a}=(M C T)_{a, b}$ e $\Phi_{c, b} \bullet(M C P)_{c}=(M C T)_{c, b}$, a soma membro a membro das três equações resulta em

$$
\begin{aligned}
& R_{a}+R_{c}-\left[(C P)_{a}+(C P)_{b}+(C P)_{c}+(C T)_{b, a}+\ldots\right. \\
& \left.\quad \ldots(C T)_{a, b}+(C T)_{b . c}+(C T)_{c, b}\right]
\end{aligned}
$$

\section{Situação 3:}

Dada a rede logística da situação 2, o que é mais vantajoso, manter a $\mathrm{UO}_{b}$ em operação ou desativá-la?

As soluções na situação 1 e na situação 2 tratam do problema (1); a questão que se coloca agora é o problema (2).

A resposta para esta questão deve ser buscada por uma simulação da rede logística resultante da desativação de $\mathrm{UO}_{b}$. Para elaborar uma simulação simples, considera-se que as receitas e os custos próprios das UO's remanescentes permanecem constantes; alteramse, contudo, os custos de transferência de cargas entre as UO's remanescentes e os fatores de transferência de margens de contribuição primária.

Com a desativação de $\mathrm{UO}_{b}$, o sistema de equações das margens de contribuição líquida da rede logística formada pelas UO's remanescentes passa a ser

$$
\begin{aligned}
& (M C L)_{a}^{\prime \prime}=(M C P)_{a}-(M C T)_{a, c}+\left[(M C T)_{c, a}-(C T)_{a, c}\right] \\
& (M C L)_{c}^{\prime \prime}=(M C P)_{c}-(M C T)_{c, a}+\left[(M C T)_{a, c}-(C T)_{c, a}\right]
\end{aligned}
$$

A soma membro a membro de ambas as equações, feitas as devidas substituições, resulta em

$$
\begin{gathered}
K^{\prime \prime}=(M C L)_{a}^{\prime \prime}+(M C L)_{c}^{\prime \prime}=R_{a}+R_{c}-\ldots \\
\quad \ldots\left[(C P)_{a}+(C P)_{c}+(C T)_{c, a}+(C T)_{a, c}\right]
\end{gathered}
$$

Este resultado corresponde à rede logística com a $\mathrm{UO}_{b}$ desativada. $\mathrm{O}$ resultado com a $\mathrm{UO}_{b}$ integrada à rede logística é dado por

$$
\begin{aligned}
K & =R_{a}+R_{c}-\left[(C P)_{a}+(C P)_{b}+(C P)_{c}+\ldots\right. \\
& \left.\ldots(C T)_{b, a}+(C T)_{a, b}+(C T)_{b, a}(C T)_{b, c}+(C T)_{c, b}\right]
\end{aligned}
$$

Subtraindo esta equação membro a membro da equação correspondente a $K$ ”, obtém-se:

$$
\begin{aligned}
& K^{\prime \prime}-K=\left[(C P)_{b}+(C T)_{b, a}+(C T)_{a, b}+(C T)_{b, c}+(C T)_{c b}\right] \ldots \\
& \ldots-\left[(C T)_{c, a}+(C T)_{a, c}\right]
\end{aligned}
$$

Este resultado pode assumir valores no intervalo $0>\left(K^{\prime \prime}-K\right) \geq 0$. 
$\left(K^{\prime \prime}-K\right)>0$ indica que os custos operacionais da rede logística com as três UO's em operação são superiores aos custos operacionais posteriores à desativação de $\mathrm{UO}_{b}$. Da perspectiva da rentabilidade econômica da rede logística, é vantajoso desativá-la. A decisão depende, contudo, de julgamentos de natureza política e estratégica que não estão no escopo deste artigo.

\section{VERIFICAÇÃO DE RESULTADOS DE UMA REDE LOGÍSTICA}

O novo método foi submetido a uma verificação de campo, com dados efetivos de uma empresa de transporte com 40 UO's -8 unidades filiais e 32 unidades agentes. Foram coletados dados referentes ao fluxo de cargas, rotas de transferência, capacidade dos armazéns, receitas e custos de coleta, distribuição, transferência e comissões. Como elemento de volume, foi adotado o mesmo usado pela empresa, o peso das cargas em toneladas, uma vez que as taxas de transporte (custo dominante no planejamento de uma rede logística) são determinadas com fundamento nesta dimensão.

Como as UO's manuseiam cargas sobre as quais incidem custos diferentes, o método teve que passar por algumas adaptações para assimilar a realidade, mas que não distorceram sua essência. Para as unidades agentes, por exemplo, os custos de coleta e distribuição dão lugar a comissões de coleta e distribuição, representadas por uma porcentagem sobre a receita da UO, constituindo-se assim em custo eminentemente variável.

\subsection{Rentabilidade econômica das UO's}

A Tabela 1 mostra a classificação das oito UO's consideradas mais rentáveis pelo critério de lucro (calculado de acordo com o método tradicional) e as oito UO's consideradas mais rentáveis pelo critério de margem de contribuição (calculada de acordo com o novo método), ambos os cálculos considerando o mesmo período de um ano.

Tabela 1: As oito UO's mais rentáveis (elaborada pelos autores)

\begin{tabular}{cc|cc}
\hline \multicolumn{2}{c|}{ Método tradicional } & \multicolumn{2}{c}{ Novo método } \\
\hline UO & Lucro $(\boldsymbol{R} \$)$ & UO & MCL $(\boldsymbol{R} \$)$ \\
\hline SP & 21.511 .135 & PA & 8.452 .894 \\
CP & 3.760 .302 & SP & 5.943 .167 \\
CB & 1.378 .010 & CB & 5.839 .631 \\
PA & 603.206 & CH & 2.463 .537 \\
BG & 442.846 & CV & 2.382 .051 \\
JS & 244.813 & LD & 1.827 .592 \\
JV & 117.413 & MA & 1.572 .297 \\
AP & $(35.159)$ & FN & 1.496 .136 \\
\hline
\end{tabular}

A Tabela 2 mostra a classificação das oito UO's consideradas menos rentáveis pelo critério de lucro (calculado de acordo com o método tradicional) e as oito UO's consideradas menos rentáveis pelo critério de margem de contribuição (calculada de acordo com o novo método), ambos os cálculos considerando o mesmo período de um ano.

Tabela 2: As oito UO's menos rentáveis (elaborada pelos autores)

\begin{tabular}{lccc}
\hline \multicolumn{2}{c}{ Método tradicional } & \multicolumn{2}{c}{ Novo método } \\
\hline UO & Lucro (R\$) & UO & MCL (R\$) \\
PF & $(496.152)$ & SO & 266.594 \\
SR & $(633.250)$ & ER & 265.335 \\
BL & $(715.388)$ & SL & 198.927 \\
MA & $(1.025 .508)$ & JV & 88.404 \\
CH & $(1.083 .321)$ & AP & 87.698 \\
LD & $(1.190 .393)$ & JS & $(2.156)$ \\
CV & $(1.471 .694)$ & BG & $(64.431)$ \\
FN & $(1.696 .531)$ & CP & $(656.522)$ \\
\hline
\end{tabular}

A Tabela 3 mostra o reflexo das discrepâncias entre os resultados fornecidos pelo método tradicional (lucro) e pelo novo método (margem de contribuição) na classificação das UO's quanto à rentabilidade econômica. Das oito UO's consideradas mais rentáveis de acordo com o método tradicional, cinco passaram para o extremo oposto, classificadas pelo novo método entre as oito menos rentáveis. Das oito UO's julgadas menos rentáveis pelo método tradicional, cinco foram alçadas pelo novo método ao grupo das oito UO's

Figura 3: Classificação das UO's quanto à rentabilidade (elaborada pelos autores)

\begin{tabular}{|c|c|c|c|c|}
\hline \multirow{2}{*}{ UO } & \multicolumn{2}{|c|}{$\begin{array}{l}\text { As oito UO'S mais } \\
\text { rentáveis }\end{array}$} & \multicolumn{2}{|c|}{$\begin{array}{c}\text { As oito UO'S menos } \\
\text { rentáveis }\end{array}$} \\
\hline & $\begin{array}{c}\text { Método } \\
\text { tradicional }\end{array}$ & $\begin{array}{l}\text { Novo } \\
\text { método }\end{array}$ & $\begin{array}{c}\text { Método } \\
\text { tradicional }\end{array}$ & $\begin{array}{l}\text { Novo } \\
\text { método }\end{array}$ \\
\hline $\mathrm{AP}$ & $8^{\circ}$. & & & $37^{\circ}$. \\
\hline BG & $5^{\circ}$. & & & $39^{\circ}$ \\
\hline BL & & & $35^{\circ}$. & \\
\hline $\mathrm{CB}$ & $3^{\circ}$. & $3^{\circ}$. & & \\
\hline $\mathrm{CH}$ & & $4^{\mathrm{o}}$ & $37^{\circ}$. & \\
\hline $\mathrm{CP}$ & $2^{\circ}$ & & & $40^{\circ}$ \\
\hline $\mathrm{CV}$ & & $5^{\circ}$ & $39^{\circ}$ & \\
\hline ER & & & & $34^{\circ}$ \\
\hline $\mathrm{FN}$ & & $8^{\circ}$ & $40^{\circ}$ & \\
\hline JS & $6^{\circ}$ & & & $38^{\circ}$ \\
\hline JV & $7^{\circ}$ & & & $36^{\circ}$ \\
\hline LD & & $6^{\circ}$ & $38^{\circ}$ & \\
\hline MA & & $7^{\circ}$ & $36^{\circ}$ & \\
\hline $\mathrm{PA}$ & $4^{\circ}$ & $1^{\mathrm{o}}$ & & \\
\hline $\mathrm{PF}$ & & & $33^{\circ}$ & \\
\hline $\mathrm{SL}$ & & & & $35^{\circ}$ \\
\hline $\mathrm{SO}$ & & & & $33^{\circ}$ \\
\hline $\mathrm{SP}$ & $1^{\circ}$. & $2^{\circ}$ & & \\
\hline SR & & & $34^{\circ}$. & \\
\hline
\end{tabular}


mais rentáveis. Isto é conseqüência das diferentes concepções de cálculo; enquanto o método tradicional atribui as receitas à UO que coleta cargas, o novo método atribui as receitas à UO que distribui cargas.

\subsection{Reconfiguração da rede logística}

Com fundamento nos resultados obtidos pelo novo método, foram elaboradas análises de possíveis melhorias da rentabilidade da rede logística, considerando algumas alternativas de reconfiguração da rede logística por meio de desativar algumas UO's menos rentáveis. As alternativas foram analisadas quanto à viabilidade econômica e à estratégica com fundamento nos resultados obtidos na verificação levada a termo de acordo com o algoritmo preconizado pelo novo método.

A Tabela 4 mostra comparativamente o resultado operacional da rede logística real e o resultado operacional da mais rentável das alternativas de reconfiguração da rede logística obtida apenas por desativar algumas UO's. Ambos os resultados foram determinados de acordo com o novo método. Os valores nas colunas ANTES e DEPOIS são expressos em reais e correspondem ao exercício de um ano. Os valores nas colunas $\% A$ e $\% D$ são dados como percentuais da receita; os valores na coluna $\Delta$ representam as diferenças $\% D$ - $\% A$ das linhas correspondentes.

$O$ resultado operacional da melhor alternativa de reconfiguração da rede logística é superior ao resultado operacional da rede logística real em $\mathrm{R} \$ 407.578,00$. Isto é, o resultado operacional da rede logística reconfigurada é cerca de $3,17 \%$ superior ao resultado operacional efetivamente proporcionado pela rede logística como esta efetivamente operou, o que corresponde a uma economia de $0,65 \%$ da receita.

\section{CONCLUSÕES}

Foi apresentado um novo método de contabilidade gerencial adequado para determinar a rentabilidade econômica de unidades operacionais - UO's - de uma empresa de transporte de carga fracionada, método es- te que utiliza o conceito de margem de contribuição como forma de eliminar as distorções oriundas da utilização do critério de rateio de custos.

Uma dificuldade para empregar o novo método para analisar uma configuração logística existente reside na coleta dos dados necessários, uma vez que boa parte deles não é fornecida pelos sistemas de registros contábeis voltados às necessidades dos métodos de custeio por absorção. A adoção do novo método implica a necessidade de uma reformulação radical destes sistemas de registros contábeis para adequá-los à nova concepção de gestão da rede logística. Uma vez implantado, contudo, o novo método oferece a vantagem de facilitar uma gestão dinâmica da rede logística, pois permite determinar de maneira simples e confiável a rentabilidade econômica das várias UO's isoladamente e sua contribuição para a rentabilidade econômica da rede logística como um todo. Além disto, com emprego de recursos acessíveis de informática, oferece condições para manter atualizado o sistema de gestão por meio de facilitar análises logicamente simples e confiáveis de alternativas de reconfiguração da rede logística em função de mudanças de custos, volumes e fluxos de cargas, assegurando assim orientação para decisões relativas à instalação de novas UO's ou desativação de algumas UO's existentes.

\section{BIBLIOGRAFIA}

Costa, R. P. (2003) Modelo econômico de rentabilidade de unidades operacionais de uma rede logística. Notas de aula do Departamento de Engenharia de Produção, Escola Politécnica da Universidade de São Paulo.

Kaplan, R. e A. Atkinson (1989) Advanced management accounting. 2nd. ed. Englewood Cliffs, NJ: Prentice Hall.

Martins, E. (1990) Contabilidade de custos. 4a . ed. São Paulo: Atlas.

Wanke, P. (2003) Aspectos fundamentais do problema de localização de instalações em redes logísticas. <http://www.coppead.ufrj.br/ fs-public.htm>, acesso em 01/07/2003.

Tabela 4: Resultado real e simulado (elaborada pelos autores)

\begin{tabular}{lccccc}
\hline \multicolumn{1}{c}{ Discriminação } & Antes & \%A & Depois & \% D & \\
\hline Receita & 62.277 .660 & 100 & 62.277 .660 & 100 & 0 \\
(-) Custos de coleta & 7.224 .478 & 11,60 & 7.198 .440 & 11,56 & -0.04 \\
(-) Comissões de coleta & 2.136 .557 & 3,40 & 2.065 .267 & 3,32 & $-0,08$ \\
(-) Custos de distribuição & 3.188 .258 & 5,12 & 3.187 .123 & 5,12 & $\approx 0$ \\
(-) Comissões de distribuição & 8.674 .349 & 13,92 & 8.649 .284 & 13,89 & $-0,03$ \\
(-) Custos fixos & 9.260 .407 & 14,87 & 9.048 .194 & 14,53 & $-0,34$ \\
(-) Custos de transferência & 18.921 .153 & 30,38 & 18.849 .315 & 30,27 & $-0,11$ \\
$=$ Resultado operacional & $\mathbf{1 2 . 8 7 2 . 4 5 8}$ & $\mathbf{2 0 , 6 7}$ & $\mathbf{1 3 . 2 8 0 . 0 3 6}$ & $\mathbf{2 1 , 3 2}$ & $\mathbf{+ 0 , 6 5}$ \\
\hline
\end{tabular}

